\title{
Effects of Cyclooxygenase Inhibitors on Neuropathic Pain following Spinal Nerve Ligation in Rats
}

\author{
*Department of Anesthesiology and Pain Medicine, Ilsan Paik Hospital, Inje University College of Medicine, Ilsan; \\ Department of Anesthesiology and Pain Medicine, Korea University College of Medicine, Seoul, Korea
}

Woon Suck Kang, M.D.*, Jang Su Park, M.D.*, Jung Won Kim, M.D.*, Suk Min Yoon, M.D. ${ }^{\dagger}$, and Ji Yong Park, M.D.

Background: The facilitatory effect of spinal prostaglandins (PGs) on nociceptive transmission suggests that early PG synthesis after nerve injury could be important in the development of allodynia.

Methods: The aim of this study is to examine the effects of diclofenac (nonselective COX inhibitor), SC-560 (selective COX-1 inhibitor), and NS-398 (selective COX-2 inhibitor) on mechanical allodynia and thermal hyperalgesia in the neuropathic pain model. The rats underwent right L5 spinal nerve ligation (SNL) and were assigned to three COX inhibitor groups to be injected intraperitoneally with different administration dosages $(0.2 \mathrm{mg}, 1 \mathrm{mg}, 5 \mathrm{mg}) 30$ minutes before, and at 1,2 , and 3 days after SNL. The withdrawal threshold of both hindpaws in response to mechanical stimulation was measured by dynamic plantar anesthesiometer and the withdrawal ratio of right to left hindpaw was calculated. The thermal stimulation applied to both hindpaws by the plantar test was calculated different administration dosages were compared with the vehicle group.

Results: There were no differences in mechanical allodynia among the lower dosage groups $(0.2 \mathrm{mg})$ until 14 days after SNL. However, $1 \mathrm{mg}$ of NS-398 decreased mechanical allodynia compared with the vehicle group at 14 days after SNL, and $5 \mathrm{mg}$ of NS-398 decreased mechanical allodynia at 3 days after SNL. However, there was no difference in thermal hyperalgesia between the groups.

Conclusions: These results suggest that intraperitoneal administration of COX inhibitor (especially selective COX-2 inhibitor) after nerve ligation injury can attenuate the development of mechanical allodynia. (Korean $\mathbf{J}$ Anesthesiol 2005; 49: S 41 6)

Key Words: cyclooxygenase inhibitor, diclofenac, neuropathic pain, NS-398, SC-560, spinal nerve ligation.

\section{INTRODUCTION}

The presence of neuropathic pain, a significant problem that diminishes the quality of life for many sufferers, is exacerbated by the lack of adequate therapy. ${ }^{1)}$ Cyclooxygenase (COX) is an enzyme that catalyzes the conversion of arachidonic acid to prostaglandins $(\mathrm{PGs})^{2}$. $\mathrm{COX}$ inhibitors are the most commonly prescribed analgesics worldwide for the management of acute pain and inflammatory pain (e.g. rheumatoid arthritis) due to their inhibition of COX, and have been used as adjunctive therapy for

Received : July 8, 2005

Corresponding to : Suk Min Yoon, Department of Anesthesiology and Pain Medicine, Korea University College of Medicine, 1 Anam-dong 5-ga, Seongbuk-gu, Seoul 136-705, Korea, Tel: 82-2-920-5772, Fax: 82-2925-5667, E-mail: ysmin@korea.ac.kr

This article is a doctoral dissertation. pain management following a variety of surgical procedures. ${ }^{3-6)}$ In the previous decade, two different COX forms were characterized: COX-1, which is expressed in a constitutive manner, and COX-2, which is highly inducible in response to inflammatory stimuli. ${ }^{7}$ Thereafter, a number of studies regarding the analgesic effects of COX-2 inhibitors have reported the effects of long-term use of COX inhibitors. ${ }^{8,9)}$

The facilitatory effect of spinal PGs on nociceptive transmission suggests that early PG synthesis after peripheral nerve injury could be important in the development of allodynia which is the neuropathic condition in which pain is evoked by a stimulus that does not normally evoke pain (e.g., cold breeze or light touch). ${ }^{10)}$ Indeed, studies of the effects of spinal PGs on normal nociceptive neurotransmission provide clues as to how this could occur. PGs ${ }^{1)}$ stimulate wide dynamic range (WDR) neurons in the rat dorsal horn, ${ }^{2,11)}$ sensitize these same neurons to noxious mechanical stimulation, ${ }^{3,12)}$ enhance glutamate and substance $\mathrm{P}$ release from 
primary afferent terminals in the spinal cord, ${ }^{4,13,14)}$ reduce glycinemediated inhibition in the spinal cord. ${ }^{15)}$ Certainly, there is growing experimental evidence linking spinal PGs to neuropathic pain. Nevertheless, it remains unclear whether COX inhibitors can alleviate neuropathic pain. Treatment with intrathecal SC-560 (selective COX-1 inhibitor) prevents the decrease in withdrawal threshold of mechanical stimulation. ${ }^{16)}$ Etodolac (selective COX-2 inhibitor) alleviates heat-evoked hyperalgesia. ${ }^{17)}$ But piroxicam (selective COX-1 inhibitor) and NS-398 (selective COX-2 inhibitor) are without significant antiallodynic effect when administered alone. ${ }^{1)}$ Therefore the aim of this study is to examine the effects of three types of COX inhibitors (nonselective COX, selective COX-1 and selective COX-2 inhibitor) on mechanical allodynia and heat hyperalgesia in the spinal nerve ligation (SNL) model in rats.

\section{MATERIALS AND METHODS}

\section{Animals}

Male Sprague-Dawley rats (weight 180-200 g, purchased from Samtako CO., Osan, Korea) were housed in groups of 4-5 in plastic cages with soft bedding in a temperature-controlled room $\left(23 \pm 1^{\circ} \mathrm{C}\right)$ illuminated from $07: 00$ to $19: 00 \mathrm{~h}$. Food and water were available ad libitum.

\section{Nerve ligation}

The L5 single nerve injury model is a modified lesion of the Kim and Chung model. ${ }^{18)}$ Briefly, rats were anesthetized with $2 \%$ enflurane in $\mathrm{O}_{2}$ delivered at a rate of $2 \mathrm{~L} / \mathrm{min}$. After surgical preparation, the rat was placed in a prone position and the right paraspinal muscles were separated from the right spinous process at the L4-S2 levels. The right L5 transverse process was carefully removed with a small rongeur to identify visually the right L4-L5 spinal nerves. The right L5 spinal nerve was isolated and tightly ligated with 5-0 silk sutures and cut approximately $1 \mathrm{~mm}$ distal to the suture.

\section{Behavior test}

The mechanical allodynia and heat hyperalgesia were assessed bilaterally. Prior to the behavior test, the rats were accustomed to the test cages for 15 minutes. The mechanical allodynia was measured by dynamic plantar anesthesiometer (Ugo Basile, Comerio, Italy). The rats were placed in a test cage with a wire mesh floor, and the tip of the anesthesiometer was applied to the middle of the plantar surface of the operated and the non-operated hindpaws.
The maximum applied force was 20 grams and all forces were applied evenly for 10 seconds. The withdrawal threshold was measured once per trial and expressed as tolerance levels in grams. Heat hyperalgesia of the hindpaws was measured using the plantar test (Ugo Basile, Comerio, Italy) as described by Hargreaves et al. ${ }^{19)}$ Rats were placed in plastic cages with a glass plate surface. The heat stimulus was applied from beneath to the middle of the plantar surface by means of a radiant heat source. Thermal sensitivity was recorded once per trial as withdrawal latency in seconds with a cut off time of 20 seconds. Both tests were repeated five times with an interval of 5 minutes. The behavioral test was carried out 1 day prior to the surgery, and at 1, 3, 7 and 14 days after the surgery.

\section{Drug administration}

The animals were randomly allocated into four experimental groups of 30 rats per group: (vehicle, diclofenac [nonselective COX inhibitor], SC-560 [selective COX-1 inhibitor] and NS-398 [selective COX-2 inhibitor]). The surgical procedure was identical in all experimental groups. The drugs were dissolved in dimethyl sulfoxide (DMSO; Sigma Chemical Co., USA) and diluted in saline. The drug injection and behavior test were done in a double-blind fashion. Each drug was injected intraperitoneally 30 minutes before the surgery and at 1,2 , and 3 days after the surgery. At thirty minutes after drug injection, the behavioral tests were assessed at 1 and 3 days after the surgery. The diclofenac, SC-560, and NS-398 groups were divided into three subgroups of 10 rats each according to the dose $(0.2,1$ and $5 \mathrm{mg} / \mathrm{kg} / \mathrm{ml})$.

\section{Statistics}

Statistical analyses were performed with SPSS 10.0 for windows (SPSS Inc., USA). The withdrawal ratios of the right to left hindpaw measured by anesthesiometer were calculated. The thermal difference of right to left hindpaw as measured by the plantar test was calculated. The results of the behavior test were presented as the mean \pm standard error of mean (SEM) ${ }^{20)}$ Repeated measure ANOVA test was used for comparison across the groups according to the time and if there was a difference, one-way ANOVA test and post-hoc comparison test were used for comparison across the groups at a corresponding time point. Statistical significance was set at $\mathrm{P}<0.05$.

\section{RESULTS}

Following SNL, mechanical allodynia and heat hyperalgesia 


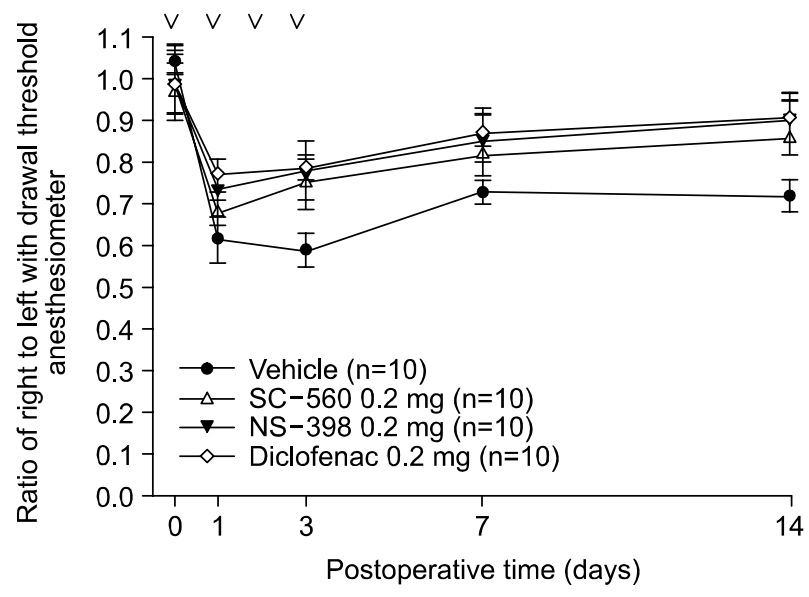

Fig. 1. Effect of intraperitoneal injection of vehicle, and $0.2 \mathrm{mg}$ of SC-560, NS-398 and diclofenac on mechanical allodynia after L5 SNL. The withdrawal ratio of injured to intact foot on mechanical allodynia (mean \pm SEM) is plotted against time.

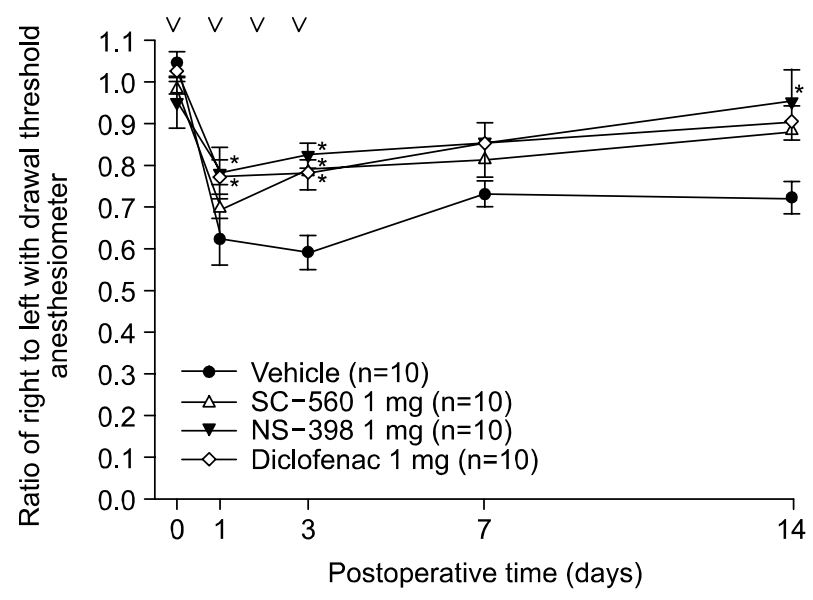

Fig. 2. Effect of intraperitoneal injection of vehicle, and $1 \mathrm{mg}$ of SC-560, NS-398 and diclofenac on mechanical allodynia after L5 SNL. The withdrawal ratio of injured to intact left foot on mechanical allodynia (Mean \pm SEM) is plotted against time. Statistically significant differences by using repeated measure ANOVA test are indicated with $\mathrm{P}<0.05$ vs. vehicle. *: indicates the time of drug injection.

were developed on 1 day and continued for 14 days. Intraperitoneal injection of a lower dose $(0.2 \mathrm{mg})$ of SC-560, NS-398 or diclofenac reduced, but not significantly, the signs of mechanical allodynia (Fig. 1). However, a medium dose (1 mg) of SC-560, NS-398 and diclofenac significantly reduced the signs of mechanical allodynia 3 days after the nerve injury, and, in the case of NS-398, and there was also a significant difference 14 days after the nerve injury (Fig. 2). In addition, in the case of higher dose (5 mg), only NS-398, but not SC-560 and diclofenac, significantly

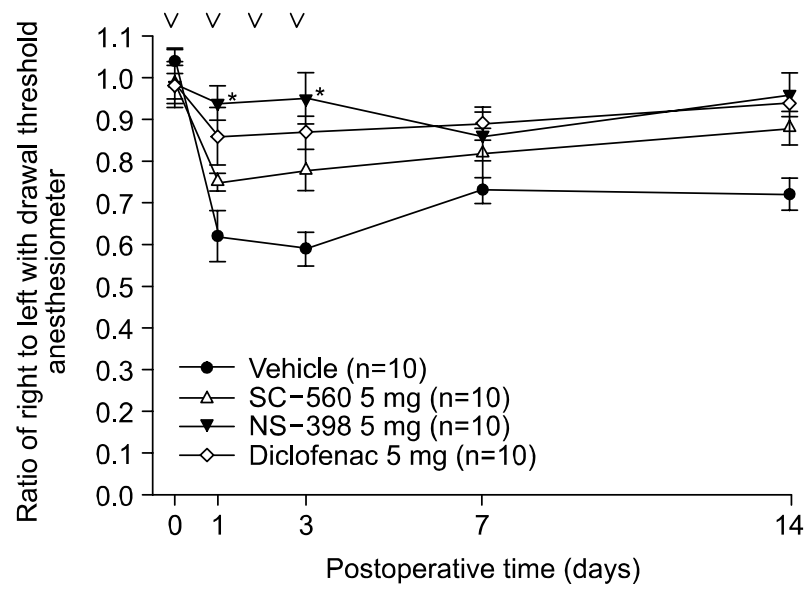

Fig. 3. Effect of intraperitoneal injection of vehicle, and $5 \mathrm{mg}$ of SC-560, NS-398 and diclofenac on mechanical allodynia after L5 SNL. The withdrawal ratio of injured to intact foot on mechanical allodynia (Mean \pm SEM) is plotted against time. Statistically significant differences by using repeated measure ANOVA test are indicated with $\mathrm{P}<0.05$ vs. vehicle. *: indicates the time of drug injection

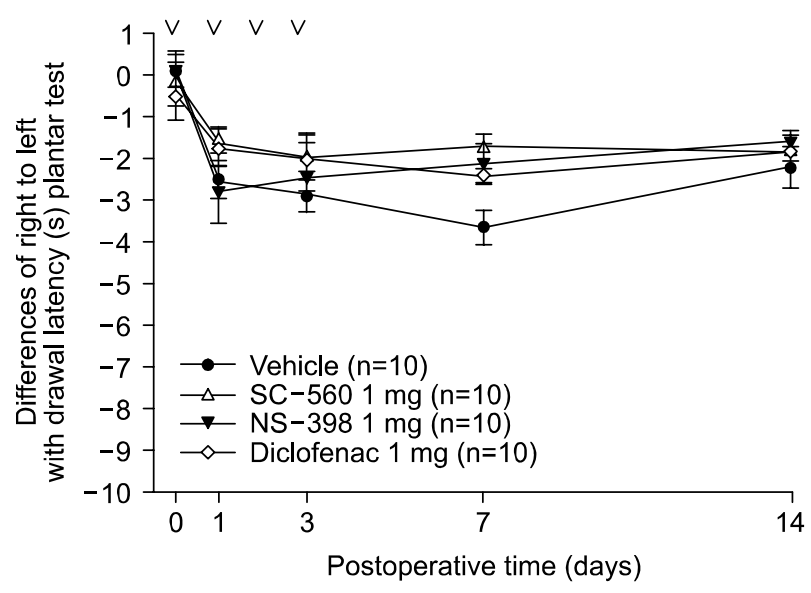

Fig. 4. Effect of intraperitoneal injection of vehicle, and $0.2 \mathrm{mg}$ of SC-560, NS-398 and diclofenac on thermal hyperalgesia after L5 SNL. The temperature difference of injured to intact foot on thermal hyperalgesia (mean \pm SEM) is plotted against time.

reduced the signs of mechanical allodynia 3 days after the nerve injury (Fig. 3). Unlike mechanical allodynia, there was no analgesic effect on the signs of heat hyperalgesia after the administration of SC-560, NS-398 or diclofenac (Fig. 4-6).

\section{DISCUSSION}

The main finding of the present study was that NS-398, a COX2-inhibitor, reduced the sign of mechanical allodynia, but not 


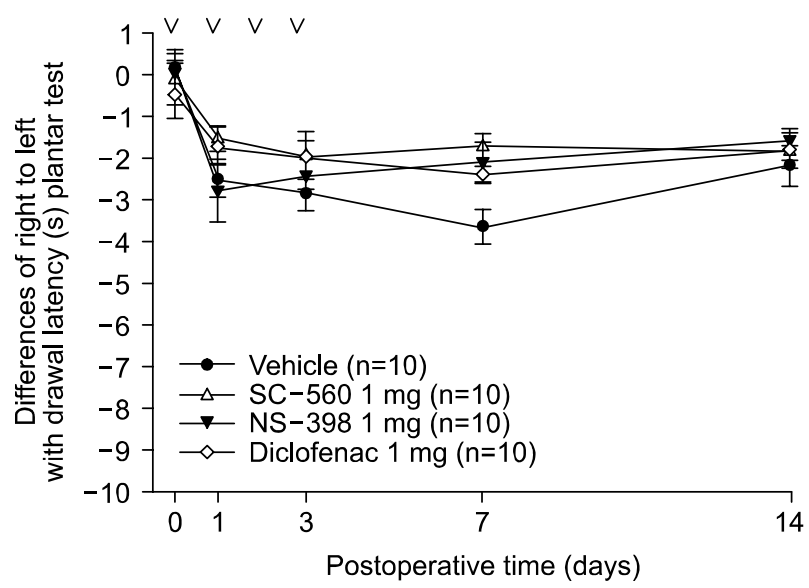

Fig. 5. Effect of intraperitoneal injection of vehicle, and $1 \mathrm{mg}$ of SC-560, NS-398, and diclofenac on thermal hyperalgesia after L5 SNL. The temperature difference of injured to intact foot on thermal hyperalgesia (mean \pm SEM) is plotted against time.

of heat hyperalgesia, at an early phase following peripheral nerve injury. The result is in line with the findings that COX-2 was expressed in the peripheral nerve, dorsal root ganglia (DRG) and thalamus at an early phase after nerve injury. ${ }^{21)}$ Taken together, spinal COX-2 probably plays an important role in the early development, but not maintenance of tactile allodynia in a rat model of neuropathic pain.

In addition, the nonselective COX inhibitor, diclofenac, attenuated mechanical allodynia at 3 days after SNL, but not beyond 4 days after SNL. COX-1 selective inhibitor, SC-560, also exhibited an antiallodynic effect. Padi and Kulkarni ${ }^{22)}$ reported that chronic systemic treatment with naproxen (non-selective COX inhibitor), but not rofecoxib (selective COX-2 inhibitor), markedly delayed the development of hypersensitivity in nerve-injured rats. They suggested that the persistent generation of PGs follows nerve injury. If we had continued with daily intraperitoneal drug injection, the antiallodynic effect of the COX inhibitors after nerve injury might have differed from the present results.

Although still controversial, increases in COX and PG in the spinal cord and peripheral nerve at an early phase after nerve injury are known to induce neuropathic pain.

One might speculate that COX inhibitors might be given as premedication before elective surgery when the patient is at risk for the development of painful neuropathies (e.g., before amputation). COX inhibitors might therefore find a place in so-called preemptive analgesia in neurosurgery. ${ }^{23)}$ It is clear that the selective COX-1 inhibitor SC-560 was less effective than the nonselective S-ibuprofen. However, because no dose-response relationship has

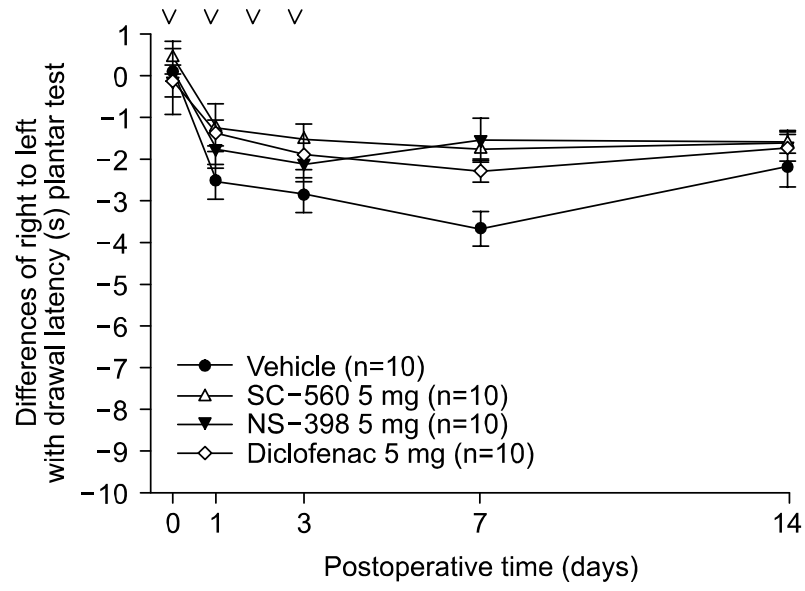

Fig. 6. Effect of intraperitoneal injection of vehicle, and $5 \mathrm{mg}$ of SC-560, NS-398, and diclofenac on thermal hyperalgesia after L5 SNL. The temperature difference of injured to intact foot on thermal hyperalgesia (mean $\pm \mathrm{SEM}$ ) is plotted against time.

been performed, this interpretation must be made with caution. ${ }^{23)}$ There are two other unresolved questions related to the pathophysiology of neuropathic pain. How do PGs promote the development of painful neuropathies, and why are they effective only earlier in the course of the disease? Under the hypothesis that PGs contribute to hyperalgesia resulting from nerve injury, Syriatowicz et $\mathrm{al}^{24)}$ showed that subcutaneous injection of SC-19220 (an EP1 PG receptor blocker) into the affected hindpaw produced significant relief of mechanical or thermal hyperalgesia and they concluded that PG E1 or E2 contribute to the peripheral mechanisms underlying hyperalgesia following nerve injury.

Two recent publications have shed light on the molecular mechanisms of PG E2 in the spinal cord. Baba et al. ${ }^{11)}$ showed that PGE2 directly depolarizes WDR neurons in the deep dorsal horn, and Ahmadi et al. ${ }^{15)}$ demonstrated that PGE2 reduces the inhibitory tone of the neurotransmitter glycine onto neurons in the superficial layers of the dorsal horn, thereby causing a disinhibition of spinal nociceptive transmission. Both mechanisms can explain why $\mathrm{PGE}_{2}$ facilitates pain sensation. Ma et $\mathrm{al}^{25)}$ suggested that following partial sciatic nerve ligation ( $\mathrm{PSNL}$ ), $\mathrm{PGE}_{2}$ is one of the possible PGs overproduced in injured nerves and that PG overproduction is involved in the up-regulation of $\mathrm{PGE}_{2}$ receptors in injured nerves. In addition, they may both contribute to plastic changes in neurotransmission between dorsal horn neurons, which may become PG-independent and largely irreversible during the disease course.

Recent evidence suggests that COX-2 expression may be involved in the development of neuropathic pain after nerve injury. 
Takahashi et $\mathrm{al}^{26)}$ demonstrated biphasic increases of COX-2 expression after L5 single spinal nerve injury. They investigated the morphological profiles of COX-2 up-regulation at an early phase (1-3 days) as well as at a late phase (7-14 days) after nerve injury. Ma and Eisenach ${ }^{27)}$ demonstrated firstly that macrophages infiltrated the damaged nerve tissue of several types of neuropathic pain models, and expressed COX-2, and secondly that inhibition of COX-2 attenuated neuropathic pain 2-4 weeks after nerve injury.

It was reported that etodolac, a COX-2 inhibitor, alleviated heat-evoked hyperalgesia in a chronic constriction injury (CCI) rat model by Suyama et al. ${ }^{17)}$ In addtion, they suggested that neuroinflammatory responses like tumor necrosis factor, IL-6 elevation and IL-1 13 cytokine gene expression in the sciatic nerve injury may be a critical mechanism of hyperalgesia in the CCI rat model. On the other hand, Zhu and Eisenach ${ }^{28)}$ showed that COX-1 expression in the spinal cord 4 days after SNL increased in the ipsilateral superficial dorsal horn with glial morphologic changes but decreased in the deep dorsal horn, ventral horn, and white matter. Therefore, they suggested that COX-1 may also be important in the maintenance of hypersensitivity after nerve injury and that targeting spinal COX-1 inhibition could relieve neuropathic pain. Four weeks after PSNL, a single intrathecal injection of the COX-1 preferring inhibitor ketorolac $(50 \mu \mathrm{g})$ significantly attenuated tactile allodynia for 6 days.

The COX-2 inhibitor NS-398 (60 $\mathrm{g})$ significantly reversed tactile allodynia at 2 hours following injection but the effect did not last longer than 24 hours, while the non-selective COX inhibitor, piroxicam (60 $\mathrm{gg}$ ) showed no effect. ${ }^{29)}$ However, indomethacin, a non-selective COX inhibitor, showed clearer inhibitory effect than selective COX-2 inhibitors (NS-398 and celecoxib) on ectopic activity recorded in both DRG and dorsal horn, ${ }^{3)}$ with more inhibition evident in the dorsal horn. ${ }^{30}$ )

The selective COX-2 inhibitors (NS-398 and celecoxib) did not generally reduce ectopic activity30) and Omana-Zapata and Bley suggested that a tonic generation of PGI2 by COX-1 could contribute to neuropathic pain. DRG cells express no COX-2, but do express COX-1, including many small diameter neurons such as calcitonin gene-related peptides (CGRP). COX-1 is also present in the spinal cord in glial cells, and is known to be activated and important in spinal sensitizing processes after peripheral nerve injury or inflammation. COX-2, not present in DRG cells, exists in spinal cord neurons, including those in the superficial dorsal horn, which is an area of known pain processing. ${ }^{31)}$

The effect of either locally or intrathecally injected COX inhibitors compared with systemic COX inhibitors suggests that efficacy may be determined firstly by the concentration achieved at the site of the nerve injury and/or in the spinal cord and secondly by the degree of COX-2 upregulation at the respective sites. Therefore, greater antinociceptive efficacy was observed when administered locally or perineurally, than when administered systemically. ${ }^{24,27,32)}$

Interestingly, Castroman and Timothy ${ }^{33)}$ reported that DMSO has a peripheral analgesic effect produced by the intravesical instillation of DMSO and that 50\% DMSO solution, but not $10 \%$, exhibits a local effect. However, we used 10\% DMSO and did not determine the analgesic effect in the nerve injury model.

At a late or chronic stage of nerve injury, mechanisms of central sensitization other than peripherally over-produced PGs may underlie the maintenance of neuropathic pain. If PG-independent allodynia developed, the antiallodynic effects of COX inhibitor were decreased. Therefore, early treatment with COX inhibitors (especially COX -2 inhibitor) in nerve-injured patients may prevent the development of intractable neuropathic pain.

The present study examined the effects of nonselective COX, selective COX-1, and selective COX-2 inhibitor on mechanical and thermal hyperalgesia by performing behavioral tests of the SNL model in rats. The results suggested that the intraperitoneal administration of selective COX-2 inhibitor (NS-398) after nerve injury can attenuate the development of mechanical allodynia but there was no effect on the signs of thermal hyperalgesia after the administration of COX inhibitors.

\section{REFERENCES}

1. Lashbrook JM, Ossipov MH, Hunter JC, Raffa RB, Tallarida RJ, Porreca F: Synergistic antiallodynic effects of spinal morphine with ketorolac and selective COX1- and COX2-inhibitors in nerve-injured rats. Pain 1999; 82: 65-72.

2. Smith WL, Marnett LJ, DeWitt DL: Prostaglandin and thromboxane biosynthesis. Pharmacol Ther 1991; 49: 153-79.

3. Barton SF, Langeland FF, Snabes MC, LeComte D, Kuss ME, Dhadda SS: Efficacy and safety of intravenous parecoxib sodium in relieving acute postoperative pain following gynecologic laparotomy surgery. Anesthesiology 2002; 97: 306-14.

4. Boni J, Korth-Bradley J, McGoldrick K, Appel A, Cooper S: Pharmacokinetic and pharmacodynamic action of etodolac in patients after oral surgery. J Clin Pharmacol 1999; 39: 729-37.

5. Gordon SM, Brahim JS, Rowan J, Kent A, Dionne RA: Peripheral prostanoid levels and nonsteroidal anti-inflammatory drug analgesia: replicate clinical trials in a tissue injury model. Clin Pharmacol Ther 2002; 72: 175-83. 
6. Immer FF, Immer-Bansi AS, Trachsel N, Berdat PA, Eigenmann V, Curatolo M: Pain treatment with a COX-2 inhibitor after coronary artery bypass operation: a randomized trial. Ann Thorac Surg 2003; 75: 490-5.

7. Feng L, Sun W, Xia Y, Tang WW, Chanmugam P, Soyoola E: Cloning two isoforms of rat cyclooxygenase: differential regulation of their expression. Arch Biochem Biophys 1993; 307: 361-8.

8. Bensen WG, Fiechtner JJ, McMillen JI, Zhao WW, Yu SS, Woods EM: Treatment of osteoarthritis with celecoxib, a cyclooxygenase-2 inhibitor: a randomized controlled trial. Mayo Clin Proc 1999; 74: 1095-105.

9. Matsumoto AK, Melian A, Mandel DR, Mcllwain HH, Borenstein D, Zhao PL: A randomized, controlled, clinical trial of etoricoxib in the treatment of rheumatoid arthritis. J Rheumatol 2002; 29: 1623-30.

10. Millan MJ: The induction of pain: an integrative review. Prog Neurobiol 1999; 57: 1-164.

11. Baba H, Kohno T, Moore KA, Woolf CJ: Direct activation of rat spinal dorsal horn neurons by prostaglandin E2. J Neurosci 2001; 21: 1750-6.

12. Willingale HL, Gardiner NJ, McLymont N, Giblett S, Grubb BD: Prostanoids synthesized by cyclo-oxygenase isoforms in rat spinal cord and their contribution to the development of neuronal hyperexcitability. Br J Pharmacol 1997; 122: 1593-604.

13. Ferreira SH, Lorenzetti BB: Intrathecal administration of prostaglandin E2 causes sensitization of the primary afferent neuron via the spinal release of glutamate. Inflamm Res 1996; 45: 499-502.

14. Hingtgen CM, Waite KJ, Vasko MR: Prostaglandins facilitate peptide release from rat sensory neurons by activating the adenosine 3',5'-cyclic monophosphate transduction cascade. J Neurosci 1995; 15: 5411-9.

15. Ahmadi S, Lippross S, Neuhuber WL, Zeilhofer HU: PGE(2) selectively blocks inhibitory glycinergic neurotransmission onto rat superficial dorsal horn neurons. Nat Neurosci 2002; 5: 3440.

16. Hefferan MP, O'Rielly DD, Loomis CW: Inhibition of spinal prostaglandin synthesis early after L5/L6 nerve ligation prevents the development of prostaglandin-dependent and prostaglandinindependent allodynia in the rat. Anesthesiology 2003; 99: 1180-8.

17. Suyama H, Kawamoto M, Gaus S, Yuge O: Effect of etodolac, a COX-2 inhibitor, on neuropathic pain in a rat model. Brain Res 2004; 1010: 144-50.

18. Kim SH, Chung JM: An experimental model for peripheral neuropathy produced by segmental spinal nerve ligation in the rat. Pain 1992; 50: 355-63.
19. Hargreaves K, Dubner R, Brown F, Flores C, Joris J: A new and sensitive method for measuring thermal nociception in $\mathrm{cu}-$ taneous hyperalgesia. Pain 1988; 32: 77-88.

20. Bingham S, Beswick PJ, Bountra C, Brown T, Campbell IB, Chessell IP: The cyclooxygenase-2 inhibitor GW406381X [2-(4ethoxyphenyl)-3-[4-(methylsulfonyl)phenyl]-pyrazolo[1,5-b]pyrida zine] is effective in animal models of neuropathic pain and central sensitization. J Pharmacol Exp Ther 2005; 312: 1161-9.

21. Zhao Z, Chen SR, Eisenach JC, Busija DW, Pan HL: Spinal cyclooxygenase-2 is involved in development of allodynia after nerve injury in rats. Neuroscience 2000; 97: 743-8.

22. Padi SS, Kulkarni SK: Differential effects of naproxen and rofecoxib on the development of hypersensitivity following nerve injury in rats. Pharmacol Biochem Behav 2004; 79: 349-58.

23. Zeilhofer HU, Brune K: A role for cyclooxygenase-1 in neuropathic pain? Anesthesiology 2003; 99: 1043-4.

24. Syriatowicz JP, Hu D, Walker JS, Tracey DJ: Hyperalgesia due to nerve injury: role of prostaglandins. Neuroscience 1999; 94: $587-94$.

25. Ma W, Eisenach JC: Four PGE2 EP receptors are up-regulated in injured nerve following partial sciatic nerve ligation. Exp Neurol 2003; 183: 581-92.

26. Takahashi M, Kawaguchi M, Shimada K, Konishi N, Furuya $\mathrm{H}$, Nakashima T: Cyclooxygenase-2 expression in Schwann cells and macrophages in the sciatic nerve after single spinal nerve injury in rats. Neurosci Lett 2004; 363: 203-6.

27. Ma W, Eisenach JC: Cyclooxygenase 2 in infiltrating inflammatory cells in injured nerve is universally up-regulated following various types of peripheral nerve injury. Neuroscience 2003; 121: 691-704.

28. Zhu X, Eisenach JC: Cyclooxygenase-1 in the spinal cord is altered after peripheral nerve injury. Anesthesiology 2003; 99: $1175-9$.

29. Ma W, Du W, Eisenach JC: Role for both spinal cord COX-1 and COX-2 in maintenance of mechanical hypersensitivity following peripheral nerve injury. Brain Res 2002; 937: 94-9.

30. Omana-Zapata I, Bley KR: A stable prostacyclin analog enhances ectopic activity in rat sensory neurons following neuropathic injury. Brain Res 2001; 904: 85-92.

31. Watkins LR, Milligan ED, Maier SF: Spinal cord glia: new players in pain. Pain 2001; 93: 201-5.

32. Ma W, Eisenach JC: Morphological and pharmacological evidence for the role of peripheral prostaglandins in the pathogenesis of neuropathic pain. Eur J Neurosci 2002; 15: 1037-47.

33. Castroman PJ, Ness TJ: Spinal neurophysiologic correlates of the analgesic actions of intravesical dimethyl sulfoxide and capsaicin in the rat. J Pain 2002; 3: 394-400. 